\title{
Big Data for Social Transportation
}

\author{
Xinhu Zheng, Wei Chen, Pu Wang, Dayong Shen, Songhang Chen, Xiao Wang, Qingpeng Zhang, \\ Liuqing Yang, Fellow, IEEE
}

\begin{abstract}
Big data for social transportation brings us unprecedented opportunities for resolving transportation problems that traditional approaches are not competent and building the next generation intelligent transportation systems. Despite that social data has been applied for transportation analysis, there remains many challenges. First, social data evolves with time and contains abundant information, posing a crucial need for data collection and cleaning. Meanwhile, each type of data has specific advantages and limitations for social transportation, and one data type alone is not capable of describing the overall state of a transportation system. Systematic data fusing approaches or frameworks for combining social signal data with different features, structures, resolutions and precisions are needed. Second, data processing and mining techniques, such as natural language processing and analysis of streaming data, require further revolutions for effectively utilizing real-time traffic information. Third, social data is connected to cyber- and physical- spaces. To address practical problems in social transportation, a suite of schemes are demanded for realizing big data in social transportation systems, like crowdsourcing, visual analysis and task-based services. In this paper, we overview data sources, analytical approaches, and application systems for social transportation, and suggest a few future research directions for this new social transportation field.
\end{abstract}

Index Terms-Big data; Social Transportation; Intelligent Transportation System; Data Analytics; Crowdsourcing.

\section{INTRODUCTION}

W ITH the fast development of sensing, computing, and networking techniques, social media and mobile devices have recently experienced a rapid growth, generating huge volumes of social signals almost in real-time. These social signals, from drivers' GPS coordinates, mobile phones' billing records to messages post on social media, record

Xinhu Zheng is with the department of Computer Science \& Engineering,University of Minnesota, Twin Cities, 55455, USA. E-mail: zheng473@umn.edu

Wei Chen is with the State Key Lab of CAD\&CG, Zhejiang University, Hangzhou, Zhejiang, 310058, China. E-mail: chenwei@cad.zju.edu.cn

$\mathrm{Pu}$ Wang is with the School of Traffic and Transportation Engineering, Central South University, Changsha, Hunan, 400083, China. E-mail: wangpu@csu.edu.cn

Dayong Shen is with the College of Information Systems and Management, National University of Defense Technology, Changsha, 410073, China E-mail: dayong.shen89@gmail.com

Songhang Chen is with the State Key Lab of Management and Control for Complex Systems, Institute of Automation, Chinese Academy of Sciences, Beijing, 100190, China. E-mail: songhang.chen@ia.ac.cn

Xiao Wang is with the State Key Lab of Management and Control for Complex Systems, Institute of Automation, Chinese Academy of Sciences, Beijing, 100190, China. E-mail: kara0807@126.com

Qingpeng Zhang is with the department of systems engineering and engineering management, City University of Hong Kong, HongKong, China E-mail: qpzhang@email.arizona.edu

Liuqing Yang is with the State Key Laboratory of Management and Control for Complex Systems, Institute of Automation, Chinese Academy of Sciences, Beijing, 100864, China. E-mail: liuqing.yang@ieee.org spatial, temporal and emotional information and establish the data foundation for social transportation research [1] - [2].

Social media and social networking platforms such as Facebook, Twitter, Weibo, and WeChat, provide ubiquitous chances for people to share ideas, emotion, and information publicly or in specialized communities, generating tremendous volumes of social signals in real-time [3]. Driven by the mobile internet services, the socialized connections among persons are made available anywhere and anytime. The collected social signals not only record the human mobility information from the mobile phones, but also encapsulate a large amount of real-time traffic information. The information can be accessed by wearable and portable devices (e.g., smart phones), and thus facilitates the usage of social signals in designing and implementing location-based services and platforms, e.g., Waze App application [4] and Uber [5]. In addition, the wide spread of social media greatly encourages the users to share location-related information online, and stimulates the wisdom of crowd on sensing a transportation system in real-time. The integrated usage of pervasive computing, social networks, and mobile internet constructs a new world of connected people, vehicles, infrastructures, and services, opening new opportunities for custom-built traffic analytics and control, data-driven intelligent transportation systems (ITS), as well as social transportation.

Traffic analysis and forecasting using social signals from mobile phones, wearable devices and social media are the typical examples of the emerging field of social transportation research [6]. Compared with works in the sociology of transportation which are typically off-line and historical, social transportation emphasizes real-time computing and embedded applications for transportation problems with on-line and interactive big data. At this earlier stage, we would suggest that new social transportation field should mainly focus in the five areas, 1) traffic or transportation analytics with big data and social signals using data mining, machine learning, and natural language processing methods; 2) crowdsourcing mechanisms for transportation based social media, social networking, and the Internet of Things (IoT) or even the Internet of Everything, especially the coming V2X, that is, vehicles to vehicles, websites, people, infrastructures communication; 3) new services beyond location-based services (LBS), such as transportation knowledge automation, especially decision-based services (DBS) or task-based services (TBS) that collecting required information in real time for transportation decisions or tasks, and information or intelligence-based services (IBS) or knowledge-based services (KBS) that recommending agents or organizations who might find the identified intelligence or knowledge useful for solving their traffic problems or improving the transportation 
performance; 4) web-based agent technology for transportation control and management, such as software robots, knowledge robots or web surrogates for traffic monitoring, safe driving, vehicular heath and energy management, at this point, the effort should be directed in developing various smart apps that collect social traffic data and link people to traffic and cars in real time; and 5) real applications and feedback for more research and development.

The desires for smart cities and smart living also provide a unique opportunity for realizing true intelligent transportation with new intelligent and mobile applications. However, to achieve the transformation of mobility and transportation for smart living in smart communities or smart cities, we need more than just technology, we need to consider and include dynamics of social organizations and cultures, and we need a comprehensive system engineering approach that accommodates and embraces diversified disciplines, fields, thinking, behaviors, and social norms. With big data and social transportation, we should be able to build a world of seamlessly connected people, infrastructures, vehicles, and services in a new era of Intelligent Transportation Systems (ITS).

In this paper, we focus on two aspects of the significance of big data in intelligent transportation systems through introducing social transportation. First, we survey the sources, types and concrete analysis approaches of social signals for transportation. Second, we investigate the crowdsourcing services, the Internet of smart vehicles and web-based agent technology in the future transportation and mobility transformation.

\section{DATA FOR SOCIAL TRANSPORTATION}

In terms of data contents, social transportation data record Time, GPS coordinates, Velocity, Accelerated Velocity, Address, Texts, Video etc. For each type of social transportation data, the recorded contents are specific to one or several aspects of human mobility, and specific to information of a person or a community. The specific features, structures, resolutions and precisions of social transportation data inherently define their advantages, limitations and application scopes. In another word, different social transportation data are suitable for solving different problems.

For examples, taxi GPS data (floating car data) have been widely used to estimate real-time travel time on roads, given that taxi GPS data have both high spatial resolution $(10 \mathrm{~m}$ coordinate errors) and temporal resolution (less than 30s). Yet, taxi GPS data are not used for estimating urban travel demand, because they only represent the origins and destinations of taxi users. Another example is the mobile phone data, which have been used for estimating travel demand. The wide spatial coverage and long observational period (months to years) of mobile phone data offer us opportunities to accurately locate the home, work, entertainment locations for a large fraction of residents. However, mobile phone data are not suitable for estimating travel time on roads, because the records are too spare (several hours a record) and temporally irregular.

The advantages and limitations of different social transportation data call for the data fusing approaches and techniques. By fusing different social signal data, predictions based on one type of data can be cross validated by another type of data, new knowledge can be mutually speculated, and the prediction accuracy, the prediction precision and the algorithm performance can be improved. However, data fusion, especially for social transportation data is still in its preliminary stage for both scientific and engineering fields. Future data fusing technologies will not only require the communication and share of data from different departments, but also need a systematical methodology on organizing and analyzing data.

Table I lists potential data sources and characteristics of available social signal data.

TABLE I

SOURCES AND CHARACTERISTICS OF AVAILABLE SOCIAL DATA.

\begin{tabular}{lll}
\hline Data & Properties and Data Types & Examples \\
\hline Vehicle & Numerical: Time, GPS coordinates, & Ships, Aircraft, \\
trajectories & Velocity, Accelerated velocity; & Automobile, \\
& Categorical: Vehicle ID, Object & Train/Metro, \\
& type, Direction, Change of direction; & Mixed, e.g., [7] \\
& Textual: Name, Origin, Destination, & \\
& Station; Social media: Bayonet & \\
& images, sound, surveillance video; & \\
\hline Incident & Numerical: Time, coordinates; & Tunnel, Highway, \\
report & Categorical: Stateful events, stateless & Road, e.g., [8] \\
& events, weather conditions, incident & \\
& types; Image/Video: images, & \\
& surveillance video; & \\
\hline Human & Numerical: Time, GPS coordinates, & Cell phones, Wifi, \\
mobility & Wifi coordinates, Velocity, & e.g., [9] \\
& Accelerated velocity, Gravity; & \\
& Categorical: Cell tower ID, Service & \\
& types; Textual: Address; & \\
\hline Social & Numerical: Time, GPS coordinates; & Facebook, Twitter, \\
Networking & Categorical: User ID , Address; & Weibo, Flickr, \\
& Textual: posts (Twitter, Weibo, & Uber, Waze, Baidu \\
& WeChat etc), SMS, IP Social media: & bicycle, \\
& posts (MMS, Voice, Twitter, Weibo, & e.g., [4][10] \\
& WeChat etc); & \\
\hline Web logs & Numerical: Time; Categorical: User & Baidu, Foursquare, \\
& ID, Check-in address; Textual: & e.g., [11] \\
& comments; & \\
\hline & &
\end{tabular}

\section{ANALYZING SOCIAL TRANSPORTATION DATA}

Data analytics in social transportation play a crucial role in ITS from three main aspects [12]. First, analyzing social transportation data can help improve the performance of ITS, discover user sentiments, and model user behavior at different scales [13]. Second, government administration, transportation departments, transit agencies, driver's licensing agencies and other departments have been increasingly taking social media as an essential platform for announcing schedule irregularities, waiting times and alarms. In this sense, a proactive information pushing mode is enabled. Third, by analyzing online social conversation data, government and transportation agencies can better understand the needs of citizens, identify trends, and ultimately resolve problems. Data analytics also allows transportation agencies to focus on critical issues by filtering out peripheral information.

From the viewpoint of information science, approaches for analyzing big data in social transportation can be roughly 


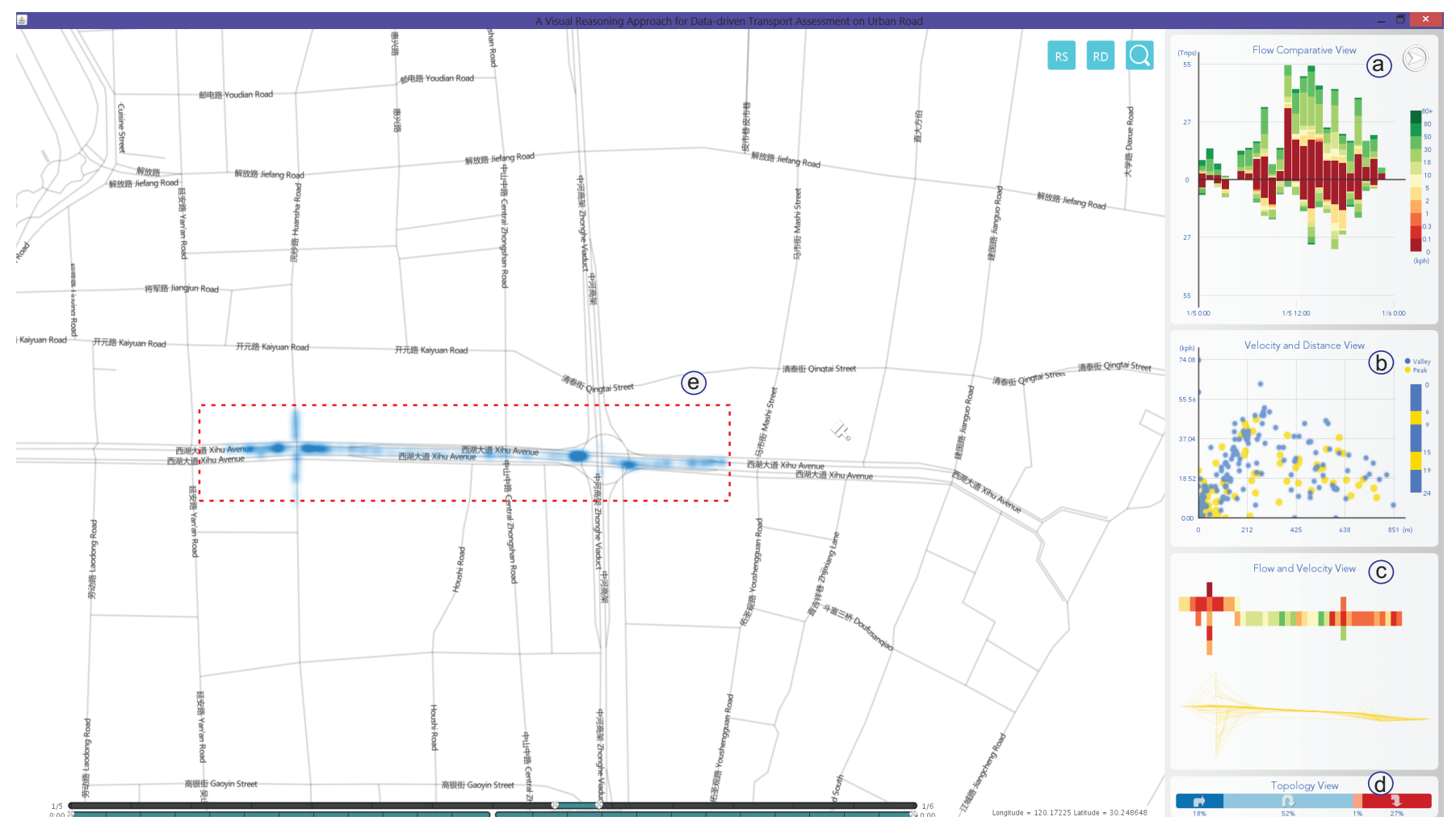

Fig. 1. A visual reasoning approach for transport assessment based on taxi trajectories [14]. The interface consists of two parts: a sketch-based query and multiple coordinated views.

classified into three categories, statistical analysis, data mining, and visual analytics.

First, statistical analysis is an analytical body of statistics that pertains to the collection, analysis, interpretation or explanation, and presentation of data. Statistical analysis methods can be not only employed to describe the statistical properties, distribution, parameters (descriptive statistics), but also employed to mine patterns in the sample data to draw inferences about the population, randomness and relationships (inferring statistics). One important branch of the statistical analysis methods is statistical physics, which uses methods of probability theory and statistics for studying large populations and approximations in solving physical problems [15][16].

Second, data mining is an interdisciplinary subfield of computer science, which denotes the computational process of discovering patterns in large data sets. Data mining lies in the intersection of artificial intelligence, machine learning, statistics, and database systems. Representative methods of data mining include clustering, classification, summarization, abnormality detection and regression analysis [17][18]. While data mining is relevant to statistical analysis, its main goal is to discover unknown and even unexpected models from data and compute the model parameters [19].

Third, visual analytics [20] is the science of analytical reasoning supported by interactive visual interfaces. In the kernel of visual analytics is data visualization, which transforms various types of data into appropriate visual representations, and greatly improves the efficiency of data understanding and analysis. Compared with statistical analysis and data mining methods, visual analytics methods allow decision makers to combine their human flexibility, creativity, and background knowledge with the enormous storage and processing capacities of high performance computing facilities to gain insight into complex problems [21]. Visual analytics can be seen as a task-driven integral approach combining visualization, human factors, and data analysis. There have been many approaches and systems that verify the values of visual analysis for social transportation. Representative systems can be found in [22][7] [23] [24] [25] [14]. Figure 1 and Figure 2 illustrate two examples of visual analysis of social transportation.

\section{TRAFFIC AND TRANSPORTATION ANALYTICS IN SOCIAL TRANSPORTATION}

Social signal data are normally characterized with big volume, wide spatial coverage, long observational period, and real-time features. The emergence of social signal data tremendously promotes our understanding of human mobility. For examples, using data collected from an online bill-tracking website, Brockmann et al. [15] analyzed the trajectories of 464,670 dollar bills to understand human mobility laws. They further used a CTRW (continuous time random walk) model to simulate human movements. González et al. [16] analyzed the trajectories of one hundred thousand mobile phone users through their billing records to uncover universal human mobility laws. Using mobile phone data, Song et al. [26] discovered that people are highly predictable regardless of their travel distance, age and sex. This finding establishes the theoretical foundation for developing accurate predictive models of human mobility. 
(a)
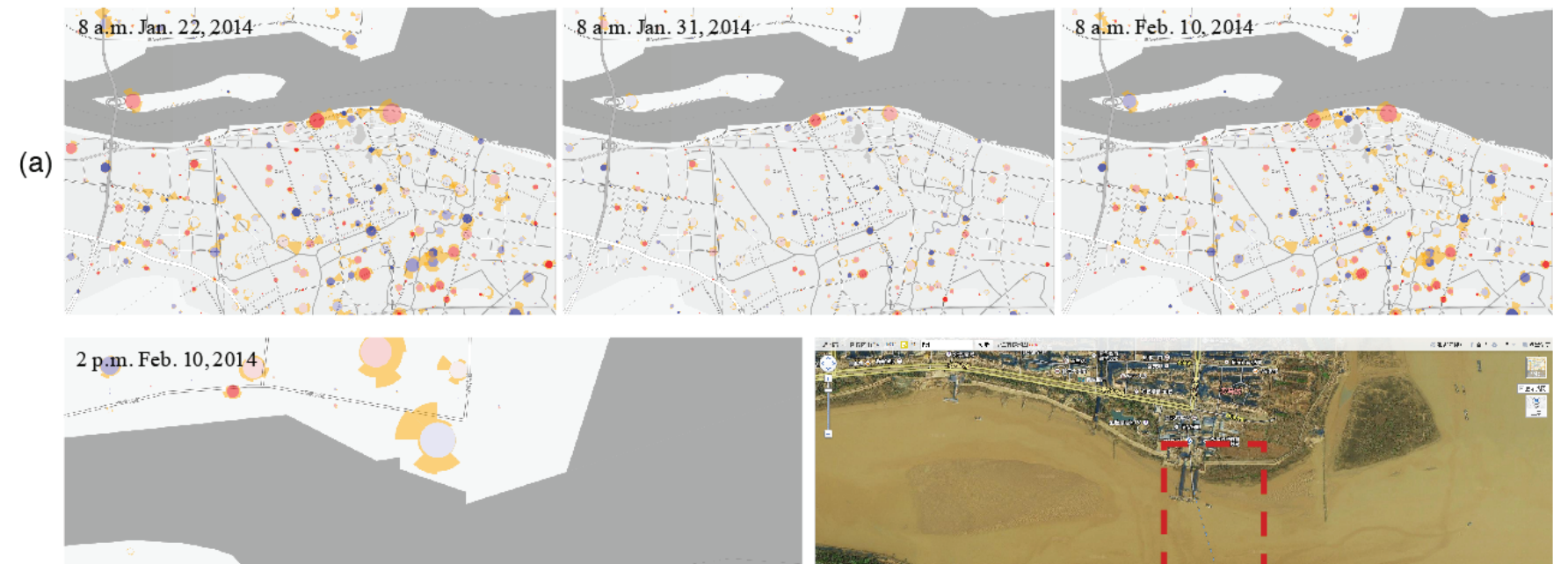

(b)
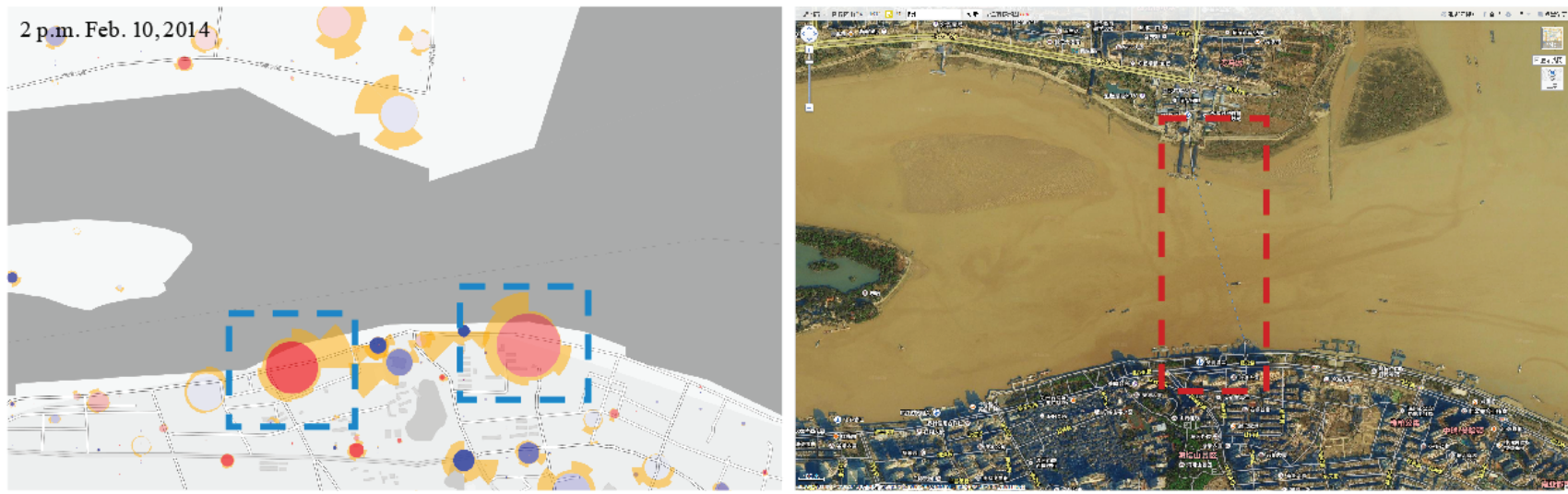

Fig. 2. Visual analysis of human mobility based on cell phone location data [23]. (a) The sunburst views for three different days; (b) The sunburst view for a ferry area and the corresponding image.

The huge developments in the area of human mobility provide us useful tools for employing social signal data in transportation analytics. Using human mobility models, origin-destination matrix (OD) can be inferred. Consequently, traffic flow can be predicted with existing traffic assignment approaches. Here, we introduce a few works on the applications of social signal data in traffic and transportation analytics.

With high spatiotemporal resolution, GPS data were widely used to estimate travel time and detect traffic congestion on roads. Using the Beijing taxi GPS data, Wang et al. [27] modeled different drivers' travel times on different road segments in different time slots with a three dimension tensor and proposed a real-time model for estimating the travel time of any path. For prototypical systems, researchers from University of California Berkeley developed a proof-of-concept traffic monitoring system in the Mobile Century project [28]. The prototype system included vehicles carrying GPS-enabled phones driving loops on a 10-mile stretch of I-880 near Union City, California. The group validated that a 2-3\% penetration of GPS-enabled cell phones in the driver population is sufficient for accurately measuring the velocity of traffic flow [28]. For practical applications, the real-time traffic flow information displayed on traffic information boards were normally inferred from taxi GPS data.

Taxi GPS data were also used for detecting traffic anomalies, building taxi sharing platforms and developing taxi calling software. Pan et al. [29] employed three months' taxi GPS data and a dataset of tweets collected from WeiBo to build a system for crowd sensing of traffic anomalies. Different from former methods, they identify anomalies according to taxi drivers' route selection behaviors. Using
New York taxi GPS data, Santi et al. [30] developed a mathematical framework to understand the tradeoff between collective benefits of taxi sharing and individual passenger discomfort. They found that with increasing but still relatively low passenger discomfort, cumulative trip length can be cut by more than $40 \%$. Now, taxi calling software have been widely adopted and made huge impacts. Examples include "UBER" in US, and "Didi Taxi", "Kuaidi Taxi" in China. These taxi calling software was all built upon real-time taxi GPS data.

The relatively low spatiotemporal resolution of mobile phone data make them not good candidates for estimating travel time on roads, but their high penetration rates and long recording periods make them good datasets for estimating OD. For examples, using mobile phone data during a long observational period, Calabrese et al. [31] proposed a method to estimate people's daily commuting OD by locating phone users' home and work locations. Wang et al. [32] employed mobile phone data for estimating the transient ODs in San Francisco Bay Area and Boston Area. There are also practical transportation applications developed with mobile phone data. In the 2010 Shanghai World Expo, a traffic prediction system based on mobile phone data was developed to infer traffic flow on roads, passenger flow on subway, and real-time congestion in Shanghai [33]. Real-time traffic information was then distributed through websites, TVs, radios, and portable devices such as mobile phones and vehicular-navigators to help travelers organize their routes in the big event.

In the public transportation system, millions of passengers keep generating huge volumes of social signals in real-time with the public transportation cards they hold. These social signals record spatiotemporal information of passengers, and have already been used to study and improve public 
transportation systems. Roth et al. [34] used the "Oyster" card data that record individual person movements in the London subway. They observed that intra urban movements are strongly heterogeneous in terms of volume, but not in terms of trip distance. Yeung et al. [35] developed a simple, principled, generic, and distributed routing algorithm capable of considering all individual path choices simultaneously. The efficacy of the proposed algorithm was tested by the "Oyster" card data. Based on the subway card data which record more than 5 million passenger trips in a typical weekday of 2013 , He et al. [36] analyzed the passenger flows distributions in the Beijing subway network and proposed a congestion avoidance routing model that can considerably reduce congestion in the subway by adjusting the routes of only a small fraction of targeted passengers.

With mobile communication devices, people can report traffic situations around them (traffic jams, traffic accidents) on ubiquitous social networking websites, such as Tweeter, Weibo and WeChat. Hence, these social networking services have became new channels for collecting traffic state information. Furthermore, the massive messages from these websites may contain information to help us understand how a traffic event is formed. Many works have recently employed social media messages in traffic and transportation analytics. For examples, Wanichayapong et al. [2] detected and classified tweets related to location and traffic information. The authors extracted traffic information from microblogs using natural language processing (NLP) methods and syntactic analysis. Schulz et al. [37] used semantic web technologies, NLP and machine learning techniques, and messages from Twitter to detect small-scale car incidents. Using online chatting messages from Tianya.cn, Wang et al. [38] used NLP approach and data mining techniques to detect traffic jams. The authors discovered people's talking point when meeting traffic jams, which can offer data support for relevant authorities to make effective decisions.

Figure 4 summarizes features, analytical approaches and applications of commonly used social data. Different types of features can be extracted from the data sources listed in Table I.

\section{CROWDSOURCING IN SOCIAL TRANSPORTATION}

Crowdsourcing is the process of solving a problem through obtaining contributions from a large group of people via online communities [39][40]. Crowdsourcing has been applied to a variety of tasks, like the DARPA Network Challenge, essay editing, biomolecule design, asteroid detection, crowd-powered search, and healthcare [41][42][43]. These tasks are usually very complicate, and cost-, time- and space-critical, which could not be achieved by a computer program, or a single person. With the wide use of smart phones and devices by people, crowdsourcing becomes a promising alternative approach to data collection and analysis. Due to its nature of the participation of large-scale people, crowdsourcing is also very suitable to be applied to the planning process of transportation systems to improve the effectiveness and feasibility [44].
As one of the most important and complicated systems in modern society, a transportation system is composed of fundamental infrastructures, transported objects, central or distributed communication, control, and computing units, as well as a human-in-the-loop feedback cycle. The main challenge for building such a complex system is how to sense the full spectrum information in a situation-aware fashion. This requirement elicits the incorporation of a crowdsourcing mechanism into ITS, for the purposes of empowering the users capabilities of sensing and controlling the transportation system and fulfilling time-critical tasks that are incompetent by traditional approaches.

Prior to the formal definition of crowdsourcing in 2006, a number of applications targeted to intelligent transportation have been successfully deployed by crowdsourcing approaches. One famous example is the OpenStreetMap [45], which is an open data project to collaboratively create an editable map of the world [46]. Traditionally, geographical information was collected by skilled, equipped, organized, and paid individuals or groups. With the availability of precise and inexpensive GPS signal receivers, a number of geographical mapping projects oriented by user participations emerged, among which OpenStreetMap is the most extensive and successful one. OpenStreetMap relies on voluntary users to contribute personally collected data for public usage (in particular, for transportation). It has been proven reliable and economic, and became an important data source other than conventional proprietary datasets [47].

Collecting and analyzing real-time or near real-time traffic information (traffic conditions, accidents, route suggestions, etc.) are equally crucial for the operations of intelligent transportation systems. Although vehicle mounted sensing and communicating devices (for Vehicle to Vehicle cooperation (V2V), Vehicle to Infrastructure cooperation (V2I) communication) are still impractical due to the cost and reliability issues, the wide adoptions of smart phones by drivers and passengers have enabled crowdsourcing as an alternative way for efficient data collection and analysis. In principle, there are two types of crowdsourcing in ITS: crowdsourcing for collecting sparse data on facilities (i.e. bike routes), and crowdsourcing for soliciting feedback on quality of transit services [44].

Crowdsourcing is a novel means for ITS, and many approaches are still in early stages for verification. Nevertheless, many pioneered works that are dedicated on crowdsourcing-based ITS have shown its great potential on revolutionizing the ITS research and developments. With the wide participating feature, crowdsourcing can be used in transportation planning, in which collective wisdom helps design efficient and user-friendly transportation systems. For instance, Waze is a GPS-based geographical navigation application which provides real-time route guide and traffic monitoring based on user-generated or shared information. The crowdsourcing mechanism makes the data collection and dynamic update ease. Another crowdsourcing service provided by Uber [5] is committed to sharing location data and transportation needs in a manner that protects the privacy of riders and drivers. The CrowdITS [48] system 
(a)

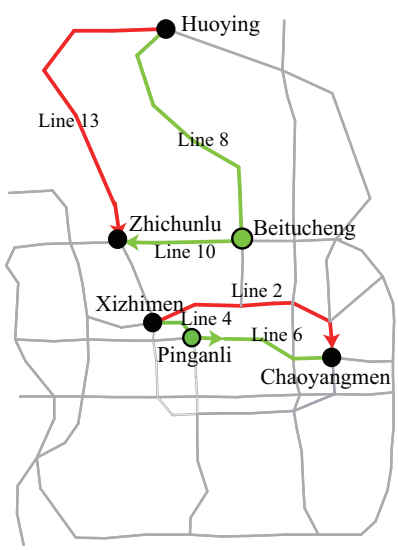

(b)

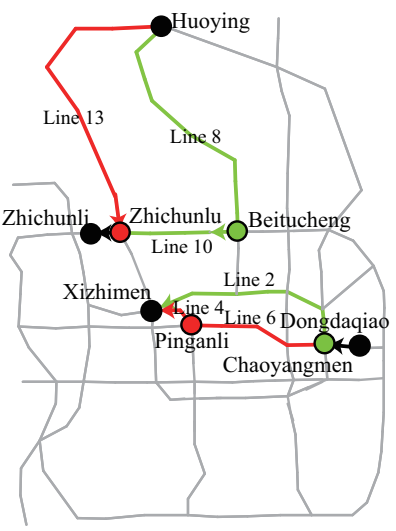

Fig. 3. Examples of minimum travel time paths (red lines) and minimum travel cost paths which take congestion cost into account (green lines) for the Beijing subway network. The black nodes indicate the origins or destinations of passenger trips, and the green and red nodes indicate the transfer stations. (a) Routing adjustments changes a direct path to a path with one transfer. (b) Routing adjustments changes the transfer stations.

Features, analytical approaches and applications of social signal data
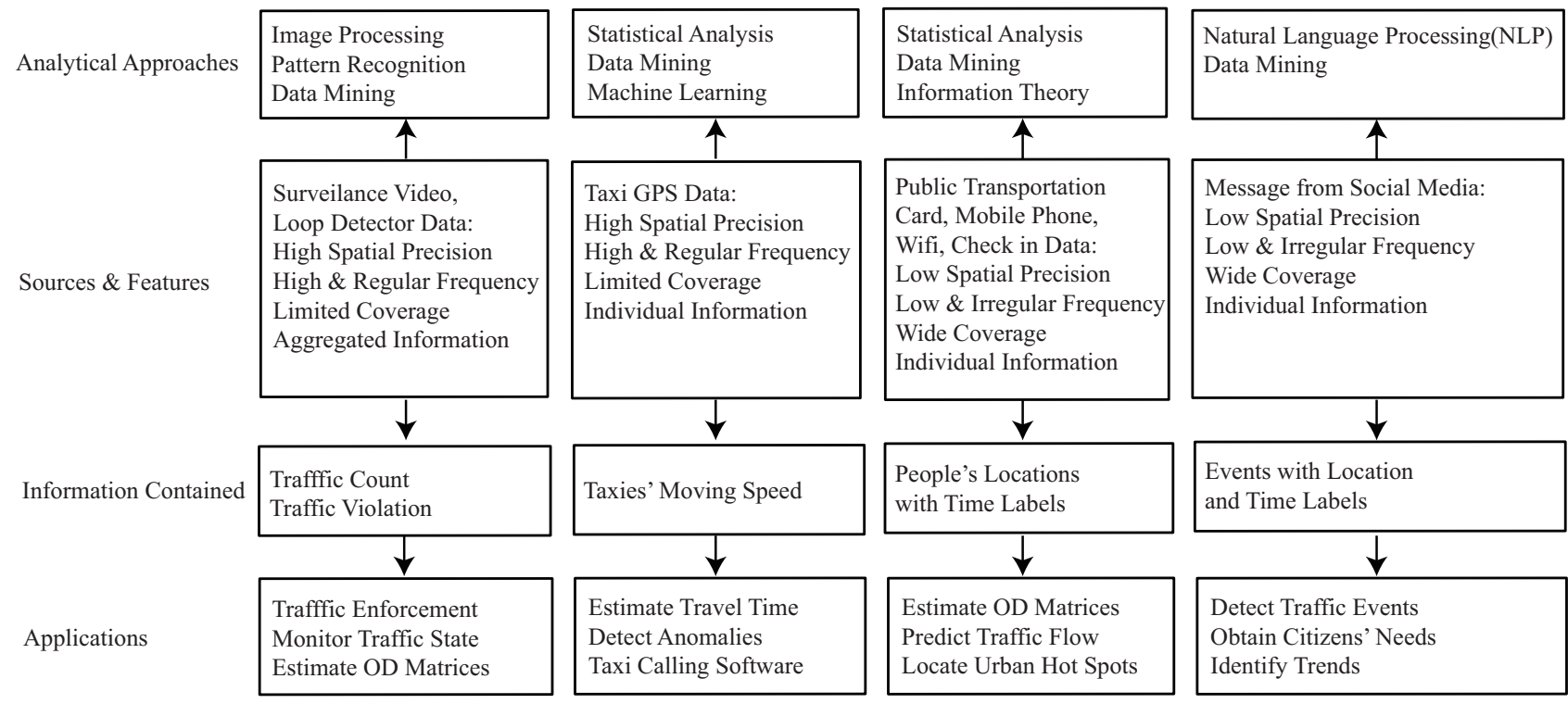

Fig. 4. The features, analytical approaches and applications of common social transportation data.

utilizes information from smart phone users for effective ITS applications. One application provides real-time traffic routing to avoid congestions by integrating the input information of drivers and selectively disseminating routing information based on geo-locations of drivers.

With the rapid growth of location-based services, wearable devices, and various types of user-generated data, we believe that crowdsourcing-enabled services will cover every component of ITS.

\section{DATA-BASED AGENTS}

Agent technology has been widely used in transportation control and management for a long time [49]. Examples include agent-based traffic signal control, agent-based transportation simulation, etc. However, there is still lack of exploration on agent technology for human-oriented transportation applications. With the development of the mobile Internet, the Internet of Vehicles and the Internet of Things, more information technologies and Artificial Intelligence technologies are used in cars to improve drivers' driving experience. For examples, Apple released Carplay to provide drivers a smarter, safer way to use iPhone during driving, and Google released Android Auto to make an android mobile device serve as a dashboard's head unit. Jaguar developed a game-like head-up display system [50] to offer drivers better driving experience. Pioneer develops Telematics to replace the rearview mirror with a display panel [51], where a built-in wide-angle camera is equipped to provide safe driving support service. Specifically, Telematics can analyze the movements of the cars in front, detect potential accidents, and provide audio and visual warnings. Under this background, web-based agent technology for transportation control and 
management is believed to be an important research direction, especially in the field of traffic monitoring, safe driving, vehicular health, and energy management. In particular, the effort should be dedicated to develop various smart mobile applications that collect social traffic data and link people to traffic and cars in real-time, like Waze and Uber.

The pioneered work in 2003 proposed a distributed framework for control and management of advanced driver assistance systems (ADAS) based on mobile agents that connect cars, homes, offices, and service centers [1]. Thereafter, there have been dramatic advances in mobile communication, robotics, and artificial intelligence, as well as the increasing public awareness and excitement on smart cars over Google's autonomous vehicles. The progress in agent technology can now be seamlessly incorporated into vehicles and auto service centers. We advocate developing task-specific agents for various driving and maintenance functions that can be hosted and executed by local platforms inside a vehicle in real-time. These agents could also move to complex remote yet powerful environments outside the vehicle, where they can be enhanced in terms of performance through further training and learning with human or computer programs. Similar ideas have been applied and verified in traffic signal control systems, where mobile-agent technology is adopted to take advantages of different algorithms to deal with dynamical changes and uncertainty in traffic and transportation environments, and achieves excellent performance beyond traditional methods [52] [53].

It is also the right time to rethink and redefine many existing vehicular hardware and software systems, such as ABS (Anti-lock Braking System), ACC (Adaptive Cruise Control), ESP (Electronic Stability Program), lane departure warning, pedestrian detection, obstacle avoidance, fatigue detection and warning, smart speed adaptation. We can consider them as vehicular robots, and enable them work in parallel with human operators and learn from driving behaviors and local environments by cloud computing. In this way, these systems can be refined over time and optimized for the best performance. Operators can effectively keep their attention on roads and enjoy a safe, smooth, and smart driving experience. Existing agent-based studies on human driving behaviors will contribute to this goal directly and significantly. Chong et al. [54] proposed an agent-based neural network model to analyze individual driver characteristics. Their experimental results show that at less than $10 \mathrm{~Hz}$ data resolution the neural agent approach outperforms the GHR model significantly and captures individual driver behavior with $95 \%$ accuracy. Abbas et al. [55] use agent based modeling techniques to model normal and safety-critical driving behaviors. For those studies that do not use the concept of agent, their findings can also be encapsulated with agents. Since agent has flexible structure and strong describing capability.

Of course, we need a more open platform and many more specified protocols in order to develop more software robots to perform diversified tasks, such as monitoring the state of drivers, checking the condition of cars, evaluating the safety of environments, and identifying available services during driving. We also need to establish reputable and reliable test beds that can thoroughly evaluate, verify, and certify these vehicular robots, so that people can create, test, and use them with confidence. Related research is now in progress. For example, a task-specific performance evaluation model has been proposed to evaluate unmanned ground vehicle (UGV) and applied in the Intelligent Vehicle Future Challenge (IVFC) annual competitions [56]. At last but not the least, security and privacy could still be a big issue for such scenarios of parallel driving. Making strict laws and policies to restrict the usage of private information could alleviate public concerns on this aspect.

\section{DATA-DRIVEN SOCIAL TRANSPORTATION SYSTEMS}

In designing and developing next generations of ITS, many new theories, technologies and systems are needed for improving traffic safety and efficiency. The key technologies for ITS are Vehicular Ad Hoc Networks (VANETs), V2I communications, traffic signal control, smart infrastructure, and intelligent vehicles. In particular, intelligent vehicles denote the ones that emerge from autonomous vehicles, such as passive or active safety systems, and driver assistance systems (e.g., lane keeping assist systems (LKAS) and stop-and-go systems).

By accessing the transportation cloud services with V2I and V2V communications, a vehicle has new expanded functions. Many studies have contributed to this direction. For instance, the cloud computing assisted ACP systems offer a cyberspace-enabled parallelism that supports a wide range of new application scenarios, such as driving in intelligent transportation spaces for integrated and traffic management, as well as vehicular safety. As a part of Parallel Transportation Management \& Control System (PtMS), a Cyber-Physical-Social Systems (CPSSs) based parallel driving system incorporates technologies of intelligent vehicles, traffic management and control, artificial transportation, and cloud computing by leveraging information from cyber-, physical-, and social-spaces, as indicated in Fig. 5.

It should be noted that human factors, human driving experiments, and behavioral analysis and modeling are critical for CPSS-based transportation systems. Special cares have to be paid on the social signals that relate to human mobility, point of interests (POIs), frequent visited destinations, and other information from mobile-based navigation devices.

A CPSS-based parallel driving system employs a layered structure, as shown in Fig. 6. In this framework, driver agents run on the controlled vehicles, and road section manager agents are installed on the roadsides or intersections. Personal agents with travel needs are included. The driver agents and personal agents can call ahead and reserve a space-time block in a road section. The transportation related CPSS conducts data fusion, computation, simulation and communication, and provides information to the Traffic Managers. The traffic managers decide whether the requested reservations are granted or rejected according to the traffic simulation results. When a request is granted, the traffic managers send the driver agents a special file for travel guidance. Otherwise, they will negotiate until the travel is arranged. 


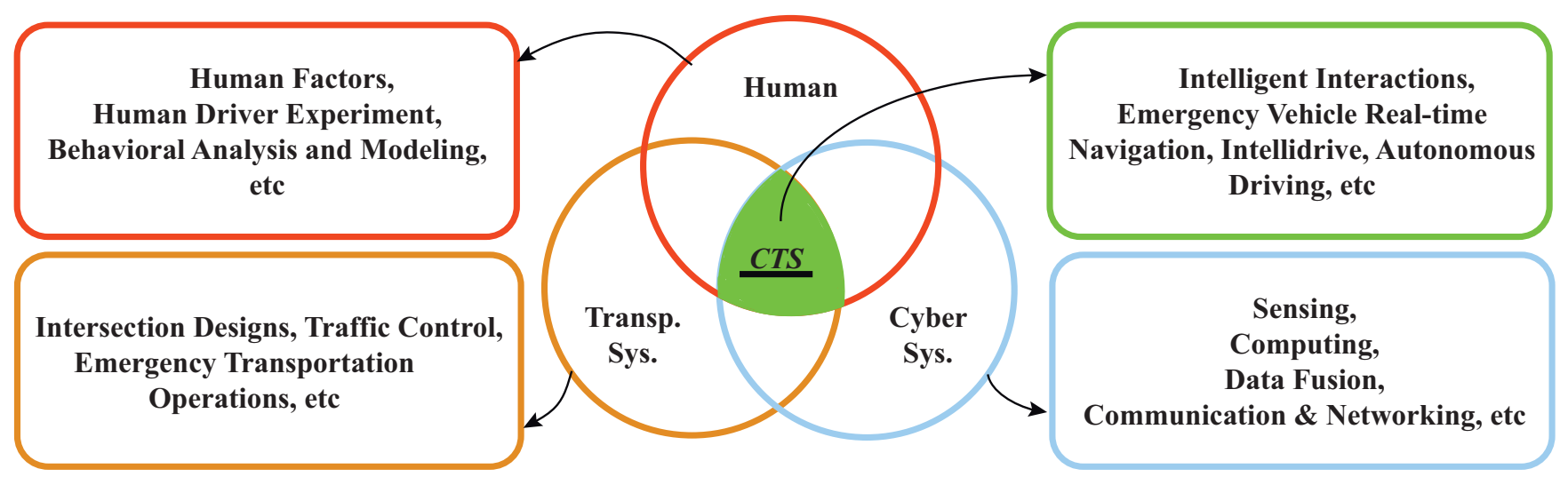

Fig. 5. Social Information for CPSS-based Transportation Systems.

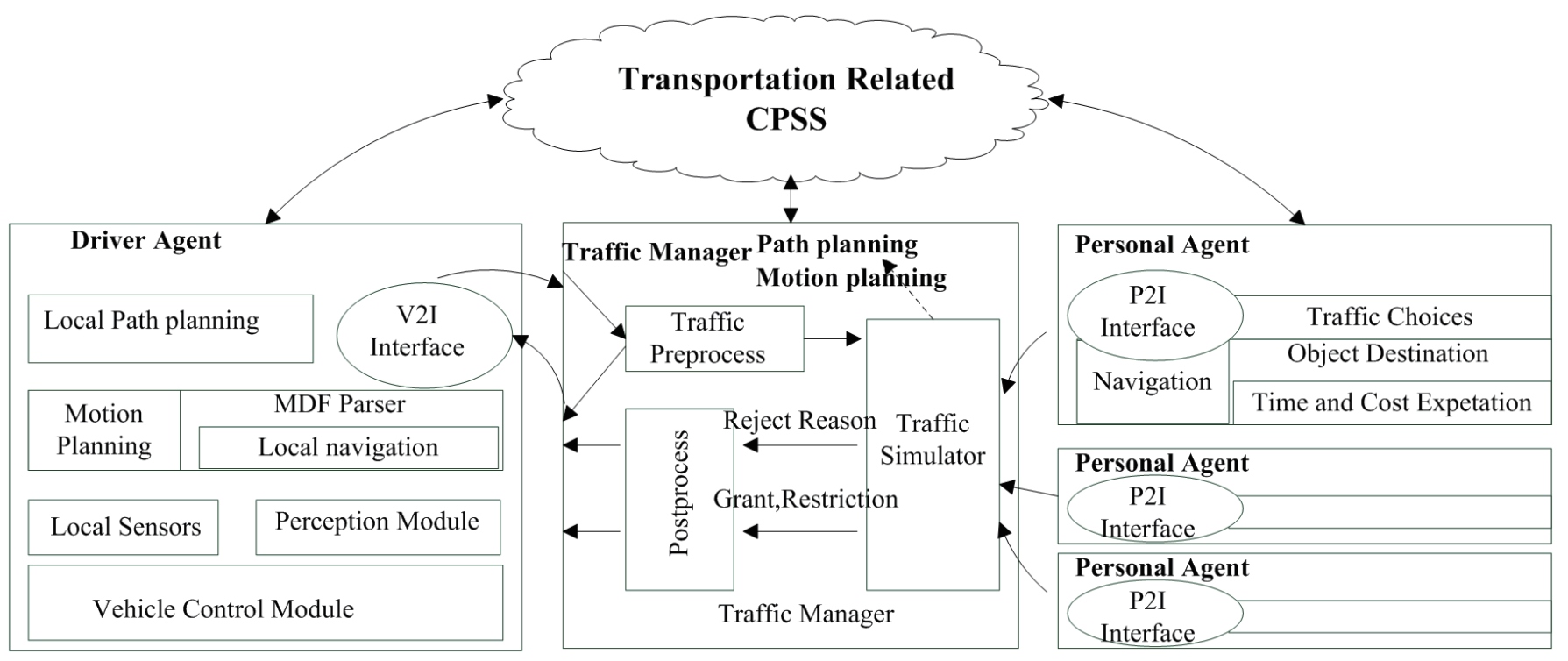

Fig. 6. Overview of CPSS-based Parallel Driving System.

The traffic managers simulate a travel with cloud computing and send driving guidance to the vehicles. This request-and-answer mechanism ensures the active control of traffic, and improves traffic safety and efficiency in complicated situations such as congested road sections and intersections. Meanwhile, there are several benefits of the communication-enabled cooperative safety applications over purely autonomous safety systems. Representative examples include: a line-of-sight detection is not required; the vehicle status can be shared to others rather than estimated by expensive vehicle mounted sensors; the cost of positioning and communication is significantly less than the autonomous sensing equipment; vehicles can coordinate maneuvers for safety goals; the movements of personal agents in critical sections like intersections are simulated to ensure the safeties.

As indicated in Fig. 6, the indispensable interface between traffic managers and driver agents as well as personal agents is the V2I/P2I link through roadside infrastructures. In CPSS-based transportation systems, to facilitate the new roles of intelligent vehicles, a key aspect is the interaction between transportation intelligent vehicles and the road management and communication infrastructures. Intelligent vehicles need to collaborate with smart roadside infrastructures [57] in order to optimize transportation related tasks, where transportation flow and communication flow are synchronized. An important trend is the massive expansion of a wireless roadside infrastructure that will establish a close interaction between data centers, mobile devices, transportation vehicles, traffic managers and the communication backbone, making the roadside infrastructures smarter.

A vehicle can access the transportation cloud services by the V2I communication channel, and extend its functions with the knowledge obtained by the parallel execution of the artificial transportation system and its real counterparts. For example, till now it is still complicated for autonomous vehicles to navigate through complex traffic environments like a crowded intersection. The transportation cloud service can be used to model and analyze the situation with previous sampling or other UGV perceptions. The result can be used to help other vehicles pass through the intersection. In this example, the problem of unknown road disturbance is addressed by means of a practical technique that utilizes a cloud-based system and the smart roadside infrastructure to get the updated information about the road. Meanwhile, 
communications with other vehicles via $\mathrm{V} 2 \mathrm{~V}$ protocols help gathering local information of the road.

The vehicles are connected to each other through V2V communication channels, and meanwhile they communicate with the cloud database. This hybrid cyber-physical platform improves ride metrics of vehicles by equipping and updating an accessible database of road-profiles. In this way, the road disturbance is no longer unknown, for which simple and fast algorithms with low computation cost are sufficient.

In the physical space, reliable and efficient communications and networking among intelligent vehicles and smart roadside infrastructures is a key enabler for the various ITS applications. As described above, CPSS-based transportation systems demand frequent and accurate information exchange for data collection and traffic management, like real-time driving guidance in CPSS-based parallel driving systems. Improved communications and networking designs are needed to guarantee the system performance with real-time, high mobility, high fidelity, and extensive information sharing features in CPSS-based transportation systems. In order to achieve improved network performance in vehicular networks, more researchers have started to consider joint V2V and V2I communications [58], [59], [60]. It has been demonstrated that vehicular networks exploiting both $\mathrm{V} 2 \mathrm{~V}$ and V2I communications provide considerably better performance than the ones based on either of them individually. Communication and networking architectures properly integrating $\mathrm{V} 2 \mathrm{~V}$ and V2I communications will be the key to solve the physical space challenges in CPSS-based transportation systems. Similar to Device-to-Device (D2D) communications in cellular networks, in vehicular networks, V2V communications can also perform as an underlay to V2I communications (which is connected to smart roadside infrastructure as a central scheduling controller). This heterogeneous architecture can significantly improve the network throughput and system capacity with effective interference management protocols [58]. Simultaneous V2V and V2V communications can also guarantee the real-time and fast information exchange among vehicles and infrastructures [61].

As a result, the objective of developing a CPSS-supported intelligent vehicle can be achieved by leveraging signals from both the physical and social spaces. This provides a continuum of options between driver in total control of the vehicle and autonomous drive, consequently guaranteeing the safety of drivers, passengers, vehicles, and infrastructures that are statistically dangerous.

\section{FUTURE OF SOCIAL TRANSPORTATION}

CPSS-based Transportation 5.0 [62], more specifically, an implementation of software-defined transportation systems, $\mathrm{O} 2 \mathrm{O}$ (online to offline and vice verse) computational transportation experiments, and parallel transportation with knowledge automation for closed-loop control and management with society-wide feedback, will be realized for real-world applications. With the help of social signals, CPSS-based Transportation 5.0 can take complete control of the traffic in both physical and cyber spaces. This vision, we believe, is the future of social transportation.
The comprehensive traffic control will provide a realistic mechanism for rapid implementation of new and exciting methods and techniques in communication and control for traffic management, especially the concept from emerging new network technology, so-called software-defined networking (SDN) and named data networks (NDN), as well as the ACP-based parallel control and management of complex systems [1][62][6][63]. In SDN, network administrators are able to manage network services through abstraction or virtualization of lower level communication functionality by decoupling the system that makes decisions about where traffic is sent (the control plane) from the underlying systems that forward traffic to the selected destination (the data plane) [64]. We believe that the complete traffic control constructs a basis for software-defined traffic networking (SDTN), which is a direct implementation of artificial transportation systems in the ACP approach. This combination greatly benefits traffic network users or customers similarly or even more than those offered by SDN for communication. As a matter of fact, SDN provides a portion of the AC functionality and does not involve steps in $\mathrm{P}$, which could lead to a closed-loop network management for better performance, as described in the ACP-based parallel systems [63].

\section{ACKNOWLEDGMENT}

Wei Chen was supported in part by National 973 Program of China (2015CB352503),the Major Program of National Natural Science Foundation of China (61232012), the National Natural Science Foundation of China (61202279), the Fundamental Research Funds for the Central Universities, and the NUS-ZJU SeSama center. Qingpeng Zhang is supported in part by CityU Start-up grant (7200399), and the Natural Science Foundation of Guangdong Province, China (2014A030313753), and the National Natural Science Foundation of China (71402157). Pu Wang is supported in part by the National Natural Science Foundation of China (No. 61473320) and the Fok Ying Tong Education Foundation (No. 141075).

\section{REFERENCES}

[1] F. Wang, "Scanning the issue and beyond: Parallel driving with software vehicular robots for safety and smartness," IEEE Transactions on Intelligent Transportation Systems, vol. 15, no. 4, pp. 1381-1387, 2014.

[2] N. Wanichayapong, W. Pruthipunyaskul, W. Pattara-Atikom, and P. Chaovalit, "Social-based traffic information extraction and classification," in 2011 11th International Conference on ITS Telecommunications (ITST). IEEE, 2011, pp. 107-112.

[3] E. D'Andrea, P. Ducange, B. Lazzerini, and F. Marcelloni, "Real-time detection of traffic from twitter stream analysis," IEEE Transactions on Intelligent Transportation Systems, pp. 1-15, 2015.

[4] Waze. http://www.waze.com. Last visit: 2015-04-01.

[5] Uber. https://www.uber.com/. Last visit: 2015-04-01.

[6] F. Wang, "Scanning the issue and beyond: Real-time social transportation with online social signals," IEEE Transactions on Intelligent Transportation Systems, vol. 15, no. 3, pp. 909-914, 2014.

[7] N. Ferreira, J. Poco, H. T. Vo, J. Freire, and C. T. Silva, "Visual exploration of big spatio-temporal urban data: A study of new york city taxi trips," IEEE Transactions on Visualization and Computer Graphics, vol. 19, no. 12, pp. 2149-2158, 2013.

[8] H. Piringer, M. Buchetics, and R. Benedik, "Alvis: Situation awareness in the surveillance of road tunnels," in 2012 IEEE Conference on Visual Analytics Science and Technology (VAST). IEEE, 2012, pp. 153-162. 
[9] W. Zeng, C.-W. Fu, S. M. Arisona, and H. Qu, "Visualizing interchange patterns in massive movement data," in Computer Graphics Forum, vol. 32, no. 3pt3. Wiley Online Library, 2013, pp. 271-280.

[10] J. Bao, Y. Zheng, and M. F. Mokbel, "Location-based and preference-aware recommendation using sparse geo-social networking data," in Proceedings of the 20th International Conference on Advances in Geographic Information Systems. ACM, 2012, pp. 199-208.

[11] L. Lins, J. T. Klosowski, and C. Scheidegger, "Nanocubes for realtime exploration of spatiotemporal datasets," IEEE Transactions on Visualization and Computer Graphics, vol. 19, no. 12, pp. 2456-2465, 2013.

[12] J. Zhang, F.-Y. Wang, K. Wang, W.-H. Lin, X. Xu, and C. Chen, "Data-driven intelligent transportation systems: A survey," IEEE Transactions on Intelligent Transportation Systems, vol. 12, no. 4, pp. 1624-1639, 2011.

[13] S. Bregman and K. E. Watkins, Best Practices for Transportation Agency Use of Social Media. CRC Press, 2013.

[14] F. Wang, W. Chen, F. Wu, Y. Zhao, H. Hong, T. Gu, L. Wang, R. Liang, and $\mathrm{H}$. Bao, "A visual reasoning approach for data-driven transport assessment on urban roads," in IEEE Conference on Visual Analytics Science and Technology, 2014, pp. 103-112.

[15] D. Brockmann, L. Hufnagel, and T. Geisel, "The scaling laws of human travel," Nature, vol. 439, no. 7075, pp. 462-465, 2006.

[16] M. C. Gonzalez, C. A. Hidalgo, and A.-L. Barabasi, "Understanding individual human mobility patterns," Nature, vol. 453, no. 7196, pp. 779-782, 2008

[17] J. Zhou, A. K. Tung, W. Wu, and W. S. Ng, "A semi-lazy approach to probabilistic path prediction in dynamic environments," in Proceedings of the 19th ACM SIGKDD international conference on Knowledge discovery and data mining. ACM, 2013, pp. 748-756.

[18] F. Zhang, N. J. Yuan, D. Wilkte, Y. Zheng, and X. Xie, "Sensing the pulse of urban refueling behavior: A perspective from taxi mobility," vol. 6 , no. 3, 2015 .

[19] J. Bao, Y. Zheng, D. Wilkie, and M. F. Mokbel, "A survey on recommendations in location-based social networks," Submitted to GeoInformatica, 2014.

[20] J. J. Thomas, Illuminating the path:[the research and development agenda for visual analytics]. IEEE Computer Society, 2005.

[21] D. A. Keim, J. Kohlhammer, G. Ellis, and F. Mansmann, Mastering the information age-solving problems with visual analytics. Florian Mansmann, 2010.

[22] N. Andrienko, G. Andrienko, and S. Rinzivillo, "Experiences from supporting predictive analytics of vehicle traffic," in Proceedings of the 2014 IEEE VIS Workshop on Visualization for Predictive Analytics. IEEE, 2014

[23] Y. Ma, T. Lin, C. Li, Z. Cao, F. Wang, and W. Chen, "Mobility viewer: A Eulerian approach for studying urban crowd flow."

[24] Z. Wang, M. Lu, X. Yuan, J. Zhang, and H. Van De Wetering, "Visual traffic jam analysis based on trajectory data," IEEE Transactions on Visualization and Computer Graphics, vol. 19, no. 12, pp. 2159-2168, 2013.

[25] Z. Wang, T. Ye, M. Lu, X. Yuan, H. Qu, J. Yuan, and Q. Wu, "Visual exploration of sparse traffic trajectory data," IEEE Transactions on Visualization and Computer Graphics, vol. 20, no. 12, 2014.

[26] C. Song, Z. Qu, N. Blumm, and A.-L. Barabási, "Limits of predictability in human mobility," Science, vol. 327, no. 5968, pp. 1018-1021, 2010

[27] Y. Wang, Y. Zheng, and Y. Xue, "Travel time estimation of a path using sparse trajectories," in Proceedings of the 20th ACM SIGKDD international conference on Knowledge discovery and data mining. ACM, 2014, pp. 25-34.

[28] S. Amin, S. Andrews, S. Apte, J. Arnold, J. Ban, M. Benko, R. M. Bayen, B. Chiou, C. Claudel, C. Claudel et al., "Mobile century using GPS mobile phones as traffic sensors: A field experiment," pp. 16-20, 2008.

[29] B. Pan, Y. Zheng, D. Wilkie, and C. Shahabi, "Crowd sensing of traffic anomalies based on human mobility and social media," in Proceedings of the 21st ACM SIGSPATIAL International Conference on Advances in Geographic Information Systems. ACM, 2013, pp. 344-353.

[30] P. Santi, G. Resta, M. Szell, S. Sobolevsky, S. H. Strogatz, and C. Ratti, "Quantifying the benefits of vehicle pooling with shareability networks," Proceedings of the National Academy of Sciences, vol. 111, no. 37, pp. 13 290-13 294, 2014.

[31] F. Calabrese, G. Di Lorenzo, L. Liu, and C. Ratti, "Estimating origin-destination flows using mobile phone location data," IEEE Pervasive Computing, pp. 36-44, 2011.
[32] P. Wang, T. Hunter, A. M. Bayen, K. Schechtner, and M. C. González, "Understanding road usage patterns in urban areas," Scientific reports, vol. 2, 2012.

[33] ITSChina. http://www.itschina.org/article.asp?articleid=2259. Last visit: 2015-05-15.

[34] C. Roth, S. M. Kang, M. Batty, and M. Barthélemy, "Structure of urban movements: polycentric activity and entangled hierarchical flows," PloS one, vol. 6, no. 1, pp. 1-8, 2011.

[35] C. H. Yeung, D. Saad, and K. M. Wong, "From the physics of interacting polymers to optimizing routes on the London underground," Proceedings of the National Academy of Sciences, vol. 110, no. 34, pp. $13717-13722,2013$

[36] K. He, J. Wang, L. Deng, and P. Wang, "Congestion avoidance routing in urban rail transit networks," in 2014 IEEE 17th International Conference on Intelligent Transportation Systems (ITSC). IEEE, 2014, pp. 200-205.

[37] A. Schulz, P. Ristoski, and H. Paulheim, "I see a car crash: Real-time detection of small scale incidents in microblogs," in The Semantic Web: ESWC 2013 Satellite Events. Springer, 2013, pp. 22-33.

[38] X. Wang, K. Zeng, X.-L. Zhao, and F.-Y. Wang, "Using web data to enhance traffic situation awareness," in 2014 IEEE 17th International Conference on Intelligent Transportation Systems (ITSC). IEEE, 2014, pp. $195-199$.

[39] A. Doan, R. Ramakrishnan, and A. Y. Halevy, "Crowdsourcing systems on the world-wide web," Communications of the ACM, vol. 54, no. 4, pp. 86-96, 2011.

[40] J. Howe, "The rise of crowdsourcing," Wired magazine, vol. 14, no. 6, pp. 1-4, 2006.

[41] J. C. Tang, M. Cebrian, N. A. Giacobe, H.-W. Kim, T. Kim, and D. B. Wickert, "Reflecting on the DARPA red balloon challenge," Communications of the ACM, vol. 54, no. 4, pp. 78-85, 2011.

[42] Q. Zhang, F.-Y. Wang, D. Zeng, and T. Wang, "Understanding crowd-powered search groups: a social network perspective," PloS one, vol. 7, no. 6, pp. 1-16, 2012.

[43] A. W. Armstrong, S. Cheeney, J. Wu, C. T. Harskamp, and C. W. Schupp, "Harnessing the power of crowds: Crowdsourcing as a novel research method for evaluation of acne treatments," American journal of clinical dermatology, vol. 13, no. 6, pp. 405-416, 2012.

[44] A. Misra, A. Gooze, K. Watkins, M. Asad, and C. A. Le Dantec, "Crowdsourcing and its application to transportation data collection and management," Transportation Research Record: Journal of the Transportation Research Board, vol. 2414, no. 1, pp. 1-8, 2014.

[45] OpenStreetMap. http://www.openstreetmap.org/. Last visit: 2015-04-01.

[46] M. Haklay and P. Weber, "Openstreetmap: User-generated street maps," Pervasive Computing, IEEE, vol. 7, no. 4, pp. 12-18, 2008.

[47] D. Zielstra and H. H. Hochmair, "Using free and proprietary data to compare shortest-path lengths for effective pedestrian routing in street networks," Transportation Research Record: Journal of the Transportation Research Board, vol. 2299, no. 1, pp. 41-47, 2012.

[48] K. Ali, D. Al-Yaseen, A. Ejaz, T. Javed, and H. S. Hassanein, "Crowdits: Crowdsourcing in intelligent transportation systems," in Wireless Communications and Networking Conference (WCNC), 2012 IEEE. IEEE, 2012, pp. 3307-3311.

[49] B. Chen and H. H. Cheng, "A review of the applications of agent technology in traffic and transportation systems," IEEE Transactions on Intelligent Transportation Systems, vol. 11, no. 2, pp. 485-497, 2010.

[50] Jaguar reveals game-like head-up display. http://www.automotive. com/news/1407-jaguar-reveals-game-like-head-up-display/. Last visit: 2015-04-24

[51] LTE-based rearview mirror telematics unit. http://telematicswire.net. Last visit: 2015-04-24.

[52] Z. J. Li, C. Chen, and K. Wang, "Cloud computing for agent-based urban transportation systems," IEEE Intelligent Systems, vol. 26, no. 1, pp. 73-79, 2011.

[53] C. Chen, S. Li, B. Chen, and D. Wen, "Agent recommendation for agent-based urban-transportation systems," IEEE Intelligent Systems, vol. 26, no. 6, pp. 77-81, 2011.

[54] L. Chong, M. M. Abbas, and A. Medina, "Simulation of driver behavior with agent-based back-propagation neural network," Transportation Research Record: Journal of the Transportation Research Board, vol. 2249, no. 1, pp. 44-51, 2011.

[55] M. M. Abbas, B. Higgs, and L. Chong, "Combined car-following and unsafe event trajectory simulation using agent based modeling techniques," in Proceedings of the 2012 Winter Simulation Conference, 2012, pp. 9-12.

[56] "Task-specific performance evaluation of ugvs: case studies at the IVFC, author=Huang, Wu Ling and Wen, Ding and Geng, J and Zheng, Nan Ning, journal=IEEE Transactions on Intelligent 
Transportation Systems, volume $=15$, number $=5$, pages $=1969-1979$, year=2014, publisher=IEEE."

[57] X. Cheng, X. Hu, L. Yang, I. Husain, K. Inoue, P. Krein, R. Lefevre, Y. Li, H. Nishi, J. Taiber, F.-Y. Wang, Y. Zha, W. Gao, and Z. Li, "Electrified vehicles and the smart grid: The ITS perspective," IEEE Transactions on Intelligent Transportation Systems, vol. 15, no. 4, pp. 1388-1404, Aug 2014.

[58] R. Zhang, X. Cheng, Q. Yao, C.-X. Wang, Y. Yang, and B. Jiao, "Interference graph-based resource-sharing schemes for vehicular networks," IEEE Transactions on Vehicular Technology, vol. 62, no. 8, pp. 4028-4039, Oct 2013.

[59] R. Zhang, X. Cheng, L. Yang, X. Shen, and B. Jiao, "A novel centralized TDMA-based scheduling protocol for vehicular networks," IEEE Transactions on Intelligent Transportation Systems, vol. 16, no. 1, pp. 411-416, Feb 2015.

[60] X. Cheng, L. Yang, and X. Shen, "D2D for intelligent transportation systems: A feasibility study," IEEE Transactions on Intelligent Transportation Systems, vol. PP, no. 99, pp. 1-10, 2015.

[61] X. Shen, X. Cheng, L. Yang, R. Zhang, and B. Jiao, "Data dissemination in VANETs: A scheduling approach," IEEE Transactions on Intelligent Transportation Systems, vol. 15, no. 5, pp. 2213-2223, Oct 2014.

[62] F. Wang, "Scanning the issue and beyond: Computational transportation and transportation 5.0," IEEE Transactions on Intelligent Transportation Systems, vol. 15, no. 5, pp. 1861-1868, 2014.

[63] - "Scanning the issue and beyond: ITS with complete traffic control," IEEE Transactions on Intelligent Transportation Systems, vol. 15 , no. 2 , pp. 457-462, 2014

[64] "SDN," software Defined Networking Transmode's approach to SDN-Enabled Packet-Optical Networking Application.

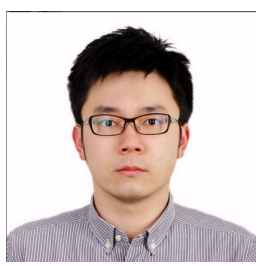

Xinhu Zheng received the B.S. degree in Control Science \& Engineering from Zhejiang University of China, in 2011 . He is currently is a graduate student in Computer Science \& Engineering at University of Minnesota, U.S.. He has published two papers in international journal and conferences. His research interests are social computing, machine learning, and data analytics.

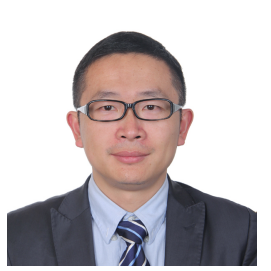

Wei Chen is a professor in State Key Lab of CAD\&CG at Zhejiang University, P.R.China. $\mathrm{He}$ has published more than 60 papers in international journal and conferences. He served as Steering committee of IEEE Pacific Visualization, Conference chair of IEEE Pacific Visualization 2015, Paper Co-chair of IEEE Pacific Visualization 2013. For more information, please refer to http://www.cad.zju.edu.cn/home/chenwei/.

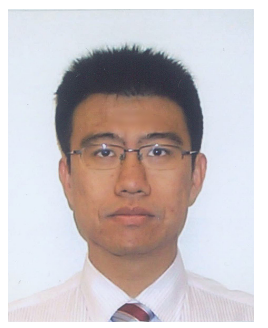

Pu Wang received the B.S. degree in Physics from University of Science and Technology of China, in 2005, and the Ph.D. degree in Physics from University of Notre Dame, in 2010. From 2010/5 to 2011/12, he worked as a Postdoc researcher in the Department of Civil and Environmental Engineering in MIT. He is currently a Full Professor of the School of Traffic and Transportation Engineering in Central South University. His research interests include complex networks, traffic analysis, human dynamics and data mining. Dr. Wang has published several papers in international leading journals, such as Science, Nature Physics, Nature Communications. He is a guest editor of IEEE Transactions on Intelligent Transportation Systems.

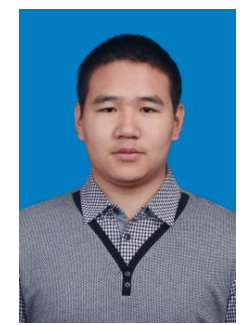

Dayong Shen received the M.Sc. degree in system engineering from National University of Defense Technology (NUDT), Changsha, P.R China in 2013. $\mathrm{He}$ is currently working toward the Ph.D degree with the Research Center for Computational Experiments and Parallel Systems at NUDT. His current research interests include web data mining, machine learning and intelligent systems.

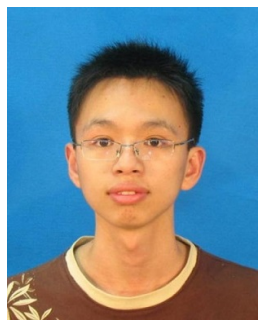

Songhang Chen was born in 1988. He received the Ph.D. degree in control theory and control engineering from the Institute of Automation, Chinese Academy of Sciences, Beijing, China, in 2014. He is currently working as an Assistant Researcher with The State Key Laboratory for Management and Control of Complex Systems, China. His research interests include travel behavior modeling, transportation simulation, and traffic signal control.

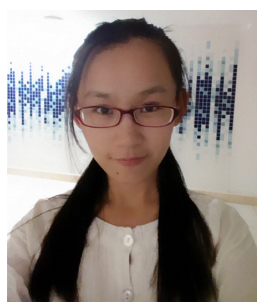

Xiao Wang received her Bachelor' $s$ degree in network engineering from Dalian University of Technology, in 2011. She is currently a Ph.D candidate in the State Key lab of Management and Control for Complex Systems, Institute of Automation, Chinese Academy of Sciences. Her research interests are social transportation, cyber movement organizations, and data analytics.

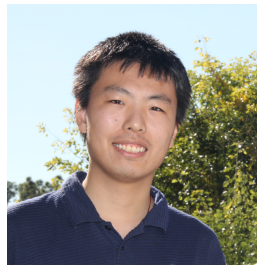

Qingpeng Zhang is an assistant professor in the department of systems engineering and engineering management at City University of Hong Kong. He received the B.S. degree in Automation from Huazhong University of Science and Technology, and the M.S. degree in Industrial Engineering and the Ph.D. degree in Systems and Industrial Engineering from The University of Arizona. Prior to joining CityU, he worked as a Postdoctoral Research Associate with The Tetherless World Constellation at Rensselaer Polytechnic Institute. His research interests include social computing, complex networks, healthcare data analytics, semantic social networks, and web science.

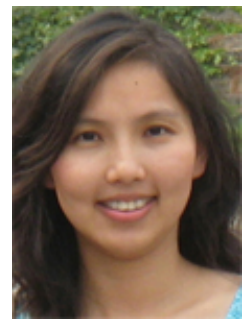

Liuqing Yang (S'02-M'04-SM'06-F'14) received her Ph.D. degree from the University of Minnesota, Minneapolis in 2004. Her general interests are in areas of communications and signal processing. Dr. Yang was the recipient of the ONR YIP award in 2007, the NSF CAREER award in 2009, the IEEE Globecom Outstanding Service Award in 2010, George T. Abell Outstanding Mid-Career Faculty Award at CSU in 2012, and Best Paper Awards at IEEE ICUWB'06, ICCC'13, ITSC'14, and Globecom'14. She has been actively serving the technical community, including organization of many IEEE international conferences, and the editorial board for a number of journals including IEEE Transactions on Communications, IEEE Transactions on Wireless Communications, and IEEE Transactions on Intelligent Transportation Systems. 